\title{
An Automata-Theoretic Model of Idealized Algol
}

\author{
Uday S. Reddy ${ }^{1}$ and Brian P. Dunphy ${ }^{2}$ \\ 1 University of Birmingham, u.s.reddy@birmingham.ac.uk \\ 2 University of Illinois at Urbana-Champaign
}

\begin{abstract}
In this paper, we present a new model of class-based Algollike programming languages inspired by automata-theoretic concepts. The model may be seen as a variant of the "object-based" model previously proposed by Reddy, where objects are described by their observable behaviour in terms of events. At the same time, it also reflects the intuitions behind state-based models studied by Reynolds, Oles, Tennent and O'Hearn where the effect of commands is described by state transformations. The idea is to view stores as automata, capturing not only their states but also the allowed state transformations. In this fashion, we are able to combine both the state-based and event-based views of objects. We illustrate the efficacy of the model by proving several test equivalences and discuss its connections to the previous models.
\end{abstract}

\section{Introduction}

Imperative programming languages provide information hiding via local variables accessible only in their declaring scope. This is exploited in object-oriented programming in a fundamental way. The use of such information hiding in everyday programming can be said to have revolutionized the practice of software development.

Meyer and Siever [13] pointed out that the traditional semantic models for imperative programs do not capture such information hiding. Rapid progress was made in the 1990's to address the problem. O'Hearn and Tennent [18] proposed a model using relational parametricity to capture the independence of data representations. Reddy [22] proposed an alternative event-based model which hides data representations entirely, and this was adapted to full Idealized Algol in [15]. Both the models have been proved fully abstract for second-order types of Idealized Algol (though this does not cover "passive" or "read-only" types) [15, 16]. Abramsky, McCusker and Honda [2, 1], refined the event-based model using games semantics and proved it fully abstract for full higher-order types.

Despite all this progress, the practical application of these models for program reasoning had stalled. As we shall see, "second-order functions" in Idealized Algol only correspond to basic functions (almost zero-order functions) in the object-oriented setting. The event-based model is a bit removed from the normal practice in program reasoning, while the applicability of the parametricity model for genuine higher-order functions has not been investigated. In fact, Pitts and 
Stark [21] showed in "awkward example" in a bare bones ML-like language, which could not be handled using the parametricity technique.

The present work began in the late 90's with the motivation of bridging the gap between state-based parametricity models and the event-based models, because they clearly had complementary strengths. These investigations led to an automata theory-inspired framework where both states and events play a role [23, $25,26]$. However, it was noticed that the basic ingredients of the model were already present in the early work of Reynolds [30]. The subsequent work focused on formalizing the category-theoretic foundations of the framework, documented in Dunphy's PhD thesis $[8,9]$, but the applications of the framework remained unexplored.

The interest in the approach has been renewed with two parallel developments in recent work. Amal Ahmed, Derek Dreyer and colleagues [3, 6, 7] began to investigate reasoning principles for higher-order ML-like languages where similar ideas have reappeared. In the application of Separation Logic to concurrency, a technique called "deny-guarantee reasoning" has been developed $[4,5]$ where, again, a combination of states and events is employed. With this paper, we hope to provide a denotational semantic foundation for these techniques and stimulate further work in this area.

\section{Framework}

The programming language we use in this paper is the language IA+ described in [27], which represents Idealized Algol [30] extended with classes.

Recall that Idealized Algol is a call-by-name simply typed lambda calculus (with full higher-order procedures including the potential for aliasing and interference), with base types supporting imperative programming. These base types include

$$
\operatorname{val}[\delta] \quad \exp [\delta] \quad \mathbf{c o m m}
$$

where $\delta$ ranges over "data types" such as int and bool.

To support classes, we use a type constructor cls so that $\operatorname{cls} \theta$ is the type of classes whose method suite is of type $\theta$. So, $\theta$ is the "interface type" of the class. The language comes with a family of predefined classes $\operatorname{Var}[\delta]$ for assignable variables of type $\delta$, whose interface type is a record type of the form:

$$
\operatorname{Var}[\delta]: \operatorname{cls}\{\text { get }: \exp [\delta], \text { put }: \operatorname{val}[\delta] \rightarrow \mathbf{c o m m}\}
$$

In essence, a variable is treated as an object with a "get" method that reads the state of the variable and "put" method that changes the state to a given value. User-defined classes are available using terms of the form

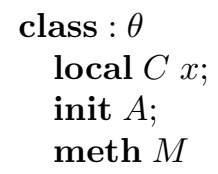


where $C$ is another class, $x$ is a locally bound identifier for the "instance variable," $A$ is a command for initializing the instance variable, and $M$ is a term of type $\theta$ serving as the method suite. For simplicity of exposition, we only consider "constant classes" in the main body of the paper, which are defined by closed terms of type $\operatorname{cls} \theta$.

Instances of classes are created in commands using terms of the form

new $C$ o. $B$

whose effect is to create an instance of class $C$, bind it to $o$ and execute a command $B$ where $o$ is allowed to occur as a free identifier. So, thinking of the binding $o . B$ as a function, new is effectively a constant of type:

$$
\operatorname{cls} \theta \rightarrow(\theta \rightarrow \operatorname{comm}) \rightarrow \operatorname{comm}
$$

This operation is an embedding, which goes to show that Idealized Algol can already express classes without this extension [27].

\section{Possible world semantics}

Semantics of Algol-like languages is normally given using a category-theoretic possible world semantics, where the "worlds" represent stores of a particular type [30]. (These are often called "store shapes." See [35, 34] for a tutorial exposition.) A simple form of worlds can be simply sets of locations [17], but it is the point of this paper to propose a more abstract treatment of stores.

We use letters $W, X, Y, Z, \ldots$ for worlds. A morphism $f: W \rightarrow X$ represents the idea that $X$ is a larger world than $W$ or, to put another way, a $W$-typed store can be extracted from an $X$-typed store via the morphism $f$. (Note the reversal of direction in the second statement.) To capture relational parametricity and data abstraction, we also assume relations between worlds, denoted as $R: W \leftrightarrow W^{\prime}$. Formally, these pieces of data should form a parametricity graph of categories [9], denoted $\mathbf{W}$.

We leave this structure unspecified for the time being, except to note that it should be able to specify for each world $W$, a set of states $\mathcal{Q}_{W}$ and a set of state transformations $\mathcal{T}_{W}$, the latter of which is made up of some collection of partial functions $\mathcal{Q}_{W} \rightarrow \mathcal{Q}_{W}$.

Each programming language type $\theta$ is interpreted as a parametricity graphfunctor (PG-functor) of the form:

$$
\llbracket \theta \rrbracket: \mathbf{W} \rightarrow \mathbf{C P O}
$$

where CPO is the category of directed-complete partial orders and continuous functions. (These functors should satisfy a special condition that they should factor through the embedding $\mathbf{C P O}_{\perp} \hookrightarrow \mathbf{C P O}$ in order to make the functor category cartesian closed [15].) We use readable notation for these functors $\llbracket \mathbf{c o m m} \rrbracket=\mathrm{COMM}, \llbracket \exp [\delta] \rrbracket=\operatorname{ExP}_{\delta}, \ldots$ The functors needed for interpreting $\mathrm{IA}+$ are shown in a schematic form in Figure 1. Note that commands for a store 


$$
\begin{aligned}
\operatorname{Comm}(X) & =\mathcal{T}_{X} \\
\operatorname{Exp}_{\delta}(X) & =\left[\mathcal{Q}_{X} \rightarrow \llbracket \delta \rrbracket\right] \\
\operatorname{VAR}_{\delta}(X) & =\operatorname{ExP}_{\delta}(X) \times[\llbracket \delta \rrbracket \rightarrow \operatorname{COMm}(X)] \\
(F \times G)(X) & =F(X) \times G(X) \\
(F \Rightarrow G)(X) & =\forall_{h: Z \leftarrow X}[F(Z) \rightarrow G(Z)] \\
(\operatorname{CLS} F)(X) & =\exists_{Z}\left(\mathcal{Q}_{Z}\right)_{\perp} \times F(Z)
\end{aligned}
$$

Fig. 1. Interpretation of IA + types

type $X$ are interpreted as state transformations for $X$, expressions for store type $X$ are interpreted as partial functions from states of $X$ to values, and variables are interpreted as pairs of get and put operations. The $\forall$ and $\exists$ operators in the last two lines are formally the parametric limit and parametric colimit constructions [9], but they can also be understood intuitively as the types of polymorphic functions and abstract types respectively. The meaning of a procedure of type $F \Rightarrow G$ at store type $X$, allows for it to be called in a larger store $Z$ (which might be obtained by allocating new local variables) and maps arguments of type $F$ in the larger store $Z$ to results of type $G$ in the store $Z$. The parametric limit interpretation of $\forall$ ensure that this will be done uniformly in the store type $Z$, depending only on the fact that it is a larger store than $X$. The interpretation of a class at a store type $X$ ignores $X$ (because we are considering only constant classes), specifies a new store $Z$ for the representation of the objects of this class, and provides an initial state and an interpretation of the methods on store $Z$. The intuitions behind this interpretation are essentially standard [18, 16, 27].

The focus of this paper is on defining a suitable category (or, rather, a parametricity graph) W for modelling stores. We do this using automata-theoretic ideas in the following sections.

\section{Stores as automata}

In algebraic automata theory $[10,11]$, we find three related notions. A semiautomaton is a triple $(Q, \Sigma, \alpha)$ where $Q$ is a set (of "states"), $\Sigma$ is a set (of "events"), $\alpha: \Sigma \rightarrow[Q \rightarrow Q]$ provides an interpretation of the events as state transformations. A more general notion is a monoid action $(Q, M, \alpha)$ where the set $\Sigma$ of events is generalized to a monoid $M$ and $\alpha$ is given to be compatible with the monoid structure. A special case of a monoid action is a transformation monoid $(Q, T)$ where $T$ is a submonoid of the monoid of state transformations $[Q \rightarrow Q]$ and, hence, $\alpha$ is implicit. In this work, we choose transformation monoids as the basis for our modelling of stores and leave its generalization to monoid actions to future work. 
The idea is to model stores as transformation monoids, whose state sets represent the states of the store (kept internal as far as possible) and whose transformations represent the actions that can be executed by commands externally. Viewed in this way, it is easy to see how the new model improves upon the earlier models. In the state-based model of [18], only the states of the store $(Q)$ are modelled. It is easy to represent sequential composition, but it is hard to represent irreversible state change. In the event-model model of [15, 22], only the events of the store $(\Sigma)$ are modelled. It is easy to represent irreversible state change, but it is hard to represent sequential composition. By using both states and events (or transformations) on an equal footing, our model combines the best features of both the traditional approaches and achieves improved accuracy.

The monoid structure of the transformation set $T$ supports sequential composition of commands. To support divergence and iteration, we require that $T$ be a monoid in $\mathbf{C P O}_{\perp}$ (the category of pointed cpo's and strict continuous functions). We refer to such a monoid as a complete ordered monoid. In addition, commands in imperative languages also have the ability to read the current state and tailor their actions accordingly. Consider if-then-else, for example. Reynolds [30] addressed this issue in his early work, and postulated an operation called the "diagonal." (However, it is more intuitive to think of it as the operation of reading the state.) Consequently, we name the resulting structures after Reynolds.

Definition 1. A Reynolds monoid is a triple $X=\left(\mathcal{Q}_{X}, \mathcal{T}_{X}\right.$, read $\left.{ }_{X}\right)$ where $\mathcal{Q}_{X}$ is a set, $\mathcal{T}_{X}$ is a submonoid of the monoid of transformations $\left[\mathcal{Q}_{X} \rightarrow \mathcal{Q}_{X}\right]$ which are jointly closed under the operation read $\operatorname{raf}_{X}\left(\mathcal{Q}_{X} \rightarrow \mathcal{T}_{X}\right) \rightarrow \mathcal{T}_{X}$ given by:

$$
\operatorname{read}_{X}(p)=\lambda x \cdot p(x)(x)
$$

The $\operatorname{read}_{X}$ operation maps a state-dependent transformation $p$ into a regular transformation, which reads the current state $(x)$, uses it to satisfy the state-dependence of $p$ and executes the resulting action. Given a transformation monoid, it is always possible to close it under the read operation by adding enough elements to $\mathcal{T}_{X}$. We call it the "read-closure" of the transformation monoid. $^{3}$

A relation of Reynolds monoids $R: X \leftrightarrow X^{\prime}$ is a pair $R=\left(R_{Q}, R_{T}\right)$

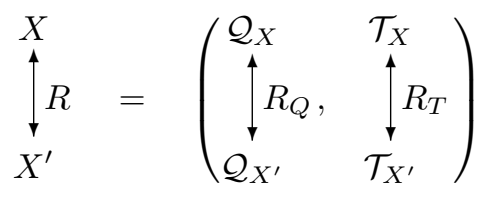

where $R_{Q}$ is a normal set-theoretic relation and $R_{T}$ is a complete ordered monoid relation (relation compatible with the units, multiplication, least elements and

\footnotetext{
${ }^{3}$ Note that the implicit monoid action $\alpha: \mathcal{T}_{X} \hookrightarrow\left[\mathcal{Q}_{X} \rightarrow \mathcal{Q}_{X}\right]$ is not part of the structure of Reynolds monoids. This is a point of departure from an earlier description of this work [28] and represents a strong generalization of the model.
} 
sup's of directed sets) such that:

$$
\operatorname{read}_{X}\left[\left[R_{Q} \rightarrow R_{T}\right] \rightarrow R_{T}\right] \operatorname{read}_{X^{\prime}}
$$

These conditions ensure that parametric transformations will include all the normal operations of imperative programming: sequential composition, if-thenelse, assignments, and iteration.

A morphism of Reynolds monoids $f: W \rightarrow X$ is a pair $f=\left(\phi_{f}, \tau_{f}\right)$ where $\phi_{f}: \mathcal{Q}_{X} \rightarrow \mathcal{Q}_{W}$ is a function and $\tau_{f}: \mathcal{T}_{W} \rightarrow \mathcal{T}_{X}$ is a complete ordered monoid morphism

$$
\stackrel{\uparrow_{W}^{X}}{\stackrel{4}{W}}=\left(\begin{array}{cc}
\mathcal{Q}_{X} & \mathcal{T}_{X} \\
\downarrow_{\phi_{f}}, & \uparrow_{\tau_{f}} \\
\mathcal{Q}_{W} & \mathcal{T}_{W}
\end{array}\right)
$$

such that the pair $\left(\left\langle\phi_{f}\right\rangle^{\smile},\left\langle\tau_{f}\right\rangle\right)$ is a relation of Reynolds monoids. The notation $\langle-\rangle$ stands for the graph of a function treated as a relation and $(-)^{\smile}$ stands for the converse of a relation.

Computationally, the intuition is that, when $X$ is a larger store than $W$, it extends and possibly constrains the states of the current world. So, it is possible to recover the state information at the level of the current world $W$ via the function $\phi_{f}$. On the other hand, the actions possible in the current store should continue to be possible in the larger store, which is modelled by the function $\tau_{f}$.

A relation-preservation square of Reynolds monoids $f[R \rightarrow S] f^{\prime}$ exists iff $\phi_{f}\left[S_{Q} \rightarrow R_{Q}\right] \phi_{f^{\prime}}$ and $\tau_{f}\left[R_{T} \rightarrow S_{T}\right] \tau_{f^{\prime}}$.

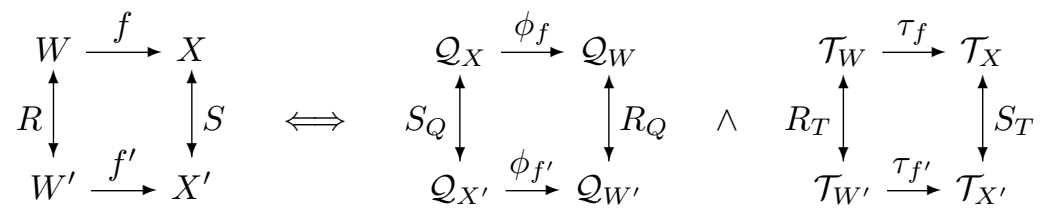

This data constitutes a cpo-enriched reflexive graph RM. (See $[9,18]$ for the background on reflexive graphs.)

To the best of our knowledge, these kinds of morphisms and relations between transformation monoids have not been studied in algebraic automata theory. The morphisms considered there generally keep the monoid of actions fixed, whereas our interest is in varying the monoid as well as the state set.

Lemma 1. RM is a cpo-enriched parametricity graph, i.e., it is relational, fibred and satisfies the identity condition.

A parametricity graph has a subsumption map whereby each morphism $f$ : $X \rightarrow Y$ is "subsumed" by an edge $\langle f\rangle: X \leftrightarrow Y$. This is given by $\langle f\rangle=\left[f, \operatorname{id}_{Y}\right] I_{Y}$. In the case of $\mathbf{R M}$, this gives $\langle f\rangle=\left(\left\langle\phi_{f}\right\rangle^{\smile},\left\langle\tau_{f}\right\rangle\right)$. 


\section{Examples of morphisms}

1. The expansion of a full transformation monoid $(Q, T(Q))$ with additional state components represented by a set $Z$, and leading to a larger world $(Q \times Z, T(Q \times Z))$, is represented by a morphism $\times Z=(\phi, \tau):(Q, T(Q)) \rightarrow$ $(Q \times Z, T(Q \times Z))$. Here, $\phi: Q \times Z \rightarrow Q$ is the projection of the $Q$ component, and $\tau: T(Q) \rightarrow T(Q \times Z)$ is given by

$$
\left.\tau(a)(q, z)=[a(q), z]_{\perp}=\text { if } a(q)=\perp \text { then } \perp \text { else }(a(q), z)\right)
$$

This example is from [30], and it is easy to verify that $\times Z$ preserves the implicit monoid action and the Reynolds diagonal.

2. A state change restriction morphism for a tm $\left(\mathcal{Q}_{X}, \mathcal{T}_{X}\right)$ restricts the state transformations to a submonoid $T^{\prime} \subseteq \mathcal{T}_{X}$. The morphism $f=(\phi, \tau)$ : $\left(\mathcal{Q}_{X}, T^{\prime}\right) \rightarrow\left(\mathcal{Q}_{X}, \mathcal{T}_{X}\right)$ is given by $\phi=\mathrm{id}_{\mathcal{Q}_{X}}$ and $\tau$ the injection of $T^{\prime}$ in $\mathcal{T}_{X}$.

3. A passivity restriction morphism is an extreme case of state change restriction morphism that prohibits all state changes: $p_{X}=\left(\operatorname{id}_{\mathcal{Q}_{X}}, \tau\right):\left(\mathcal{Q}_{X}, \mathbf{0}_{X}\right) \rightarrow$ $\left(\mathcal{Q}_{X}, \mathcal{T}_{X}\right)$ where $\mathbf{0}_{X}$ is the complete ordered monoid containing the unit transformation null $\mathcal{Q}_{X}$ and all its approximations.

\subsection{Symmetric Monoidal Structure}

Suppose $X=\left(\mathcal{Q}_{X}, \mathcal{T}_{X}\right)$ and $Y=\left(\mathcal{Q}_{Y}, \mathcal{T}_{Y}\right)$ are Reynolds monoids denoting two separate stores of locations (along with allowed transformations). We would like to define a product $\operatorname{rtm} X \star Y$ that corresponds to their combined store. There are two separate ways of doing this, depending on what transformations are allowed on the combined store. The "independent product", denoted $X \otimes Y$, allows the two parts of the store to be used independently, with no transfer of information between them. The "dependent product", denoted $X \star Y$, allows information to be transferred between them.

Independent product Given transformations $a \in \mathcal{T}_{X}$ and $b \in \mathcal{T}_{Y}$, we use the notation $a \otimes b$ for the transformation in $T\left(\mathcal{Q}_{X} \times \mathcal{Q}_{Y}\right)$ defined by:

$(a \otimes b)(x, y)=[a(x), b(y)]_{\perp}=$ if $a(x)=\perp \vee b(y)=\perp$ then $(\perp, \perp)$ else $(a(x), b(y))$

Let $\mathcal{T}_{X} \otimes \mathcal{T}_{Y}$ denote the monoid of all transformations of the form $a \otimes b$. Then, the independent product of $X$ and $Y$ is defined as

$$
X \otimes Y=\left(\mathcal{Q}_{X} \times \mathcal{Q}_{Y}, \mathcal{T}_{X} \otimes \mathcal{T}_{Y}\right)
$$

This is a transformation monoid, but not a Reynolds monoid.

Dependent product The dependent product $X \star Y$ is defined by:

$$
\begin{aligned}
\mathcal{T}_{X} \star \mathcal{T}_{Y} & =\text { the read-closure of } \mathcal{T}_{X} \otimes \mathcal{T}_{Y} \\
X \star Y & =\left(\mathcal{Q}_{X} \times \mathcal{Q}_{Y}, \mathcal{T}_{X} \star \mathcal{T}_{Y}\right)
\end{aligned}
$$


While $\mathcal{T}_{X} \otimes \mathcal{T}_{Y}$ represents an independent product of the two stores, its readclosure adds transformations of the form $\lambda(x, y) . a(x, y) \otimes b(x, y)$, allowing transfer of information between the two stores.

The corresponding relational action $R \star S: X \star Y \leftrightarrow X^{\prime} \star Y^{\prime}$ is a bit involved. The state set component is the expected one: $(R \star S)_{Q}=R_{Q} \times S_{Q}$. The transformation component is defined as follows:

$$
\begin{aligned}
& t\left[(R \star S)_{T}\right] t^{\prime} \Longleftrightarrow \\
& \forall x, x^{\prime}, y, y^{\prime} \cdot(x, y)\left[(R \star S)_{Q}\right]\left(x^{\prime}, y^{\prime}\right) \Longrightarrow \\
& \left(\exists a \in \mathcal{Q}_{X}, a^{\prime} \in \mathcal{Q}_{X^{\prime}}, b \in \mathcal{Q}_{Y}, b^{\prime} \in \mathcal{Q}_{Y^{\prime}} .\right. \\
& \quad a\left[R_{T}\right] a^{\prime} \wedge b\left[S_{T}\right] b^{\prime} \wedge \\
& \left.\quad t(x, y)=(a \otimes b)(x, y) \wedge t^{\prime}\left(x^{\prime}, y^{\prime}\right)=\left(a^{\prime} \otimes b^{\prime}\right)\left(x^{\prime}, y^{\prime}\right)\right)
\end{aligned}
$$

This says essentially that $t$ and $t^{\prime}$ can be decomposed as $a \otimes b$ and $a^{\prime} \otimes b^{\prime}$ respectively, and the corresponding components are related by $R_{T}$ and $S_{T}$. However, the choice of the witnesses $a, a^{\prime}, b$ and $b^{\prime}$ can depend on the initial states. We can make this dependence explicit by writing $a_{x y}, a_{x^{\prime} y^{\prime}}^{\prime}$ etc. instead of simple variables $a, a^{\prime}$.

The dependent product has a unit, representing the "empty store," is $\mathbf{I}=$ $\left(\mathbf{1}, \mathbf{0}_{\mathbf{1}}\right)$, where $\mathbf{0}_{\mathbf{1}}=\left\{\right.$ null $\left._{\mathbf{1}}, \bar{\perp}\right\}$. This is in fact the initial object in the parametricity graph $\mathbf{R M}$.

Hence we have the injections $\left(\iota_{1}\right)_{X, Y}: X \rightarrow X \star Y$ and $\left(\iota_{2}\right)_{X, Y}: Y \rightarrow X \star Y$ that are parametric in $X$ and $Y$. For example,

$$
\begin{gathered}
\left(\iota_{1}\right)_{X, Y}=\left(\pi_{1}: \mathcal{Q}_{X} \times \mathcal{Q}_{Y} \rightarrow \mathcal{Q}_{X}, \iota_{1}: \mathcal{T}_{X} \rightarrow \mathcal{T}_{X} \star \mathcal{T}_{Y}\right) \\
\text { where } \iota_{1}(a)=a \otimes \text { null }_{\mathcal{Q}_{Y}}
\end{gathered}
$$

\section{Semantics}

As noted in Section 2, the semantics of the programming language is given in the functor category $\mathbf{W} \rightarrow \mathbf{C P O}$ where $\mathbf{W}$ is a parametricity graph of worlds. We now choose $\mathbf{W}=\mathbf{R M}$, the parametricity graph of Reynolds monoids.

The interpretation of types is as in Fig. 1. We state the formal meaning of the parametric limit and colimit constructions appearing in the table.

The definition $(F \Rightarrow G)(X)=\forall_{h: Z \leftarrow X}[F(Z) \rightarrow G(Z)]$ uses the parametric limit, which consists of families of the form $\left\{t_{h} \in[F(Z) \rightarrow G(Z)]\right\}_{h: X \rightarrow Z}$ that are parametric in the sense that, whenever $h\left[I_{X} \rightarrow S\right] h^{\prime}$, we have $t_{h}[F(S) \rightarrow G(S)]$ $t_{h^{\prime}}$. Since $F$ and $G$ are PG-functors, such families are automatically natural [9]. It can be verified that the parametric limit is a pointed cpo under the componentwise ordering. The corresponding relation action is $(F \Rightarrow G)(R)=\forall_{S \leftarrow R}[F(S) \rightarrow$ $G(S)]$, which relates two families $\left\{t_{h}\right\}_{h: X \rightarrow Z}$ and $\left\{t_{h^{\prime}}^{\prime}\right\}_{h^{\prime}: X^{\prime} \rightarrow Z^{\prime}}$ iff, for all relations $S: Z \leftrightarrow Z^{\prime}$ and all $h, h^{\prime}$ of appropriate types, $h[R \rightarrow S] h^{\prime} \Longrightarrow$ $t_{h}[F(S) \rightarrow G(S)] t_{h^{\prime}}^{\prime}$.

The notation $\exists_{Z} T(Z)$ stands for the "parametric colimit," which is a quotient of $\coprod_{Z} T(Z)$ under the transitive closure of the similarity relation " $\sim$ ", which is 
defined by the rule:

$$
S: Z \leftrightarrow Z^{\prime} \wedge a[T(S)] a^{\prime} \Rightarrow\langle Z, a\rangle \sim\left\langle Z^{\prime}, a^{\prime}\right\rangle
$$

The equivalence class of $\langle Z, a\rangle$ under $\sim^{*}$ is denoted by $\langle Z, a\rangle$ and we call such an entity a "package." The relation $\exists_{S} T(S)$ relates two packages $\langle Z, a\rangle$ and $\left\langle Z^{\prime}, a^{\prime}\right\rangle$ iff there exists a relation $S: Z \rightarrow Z^{\prime}$ such that $a[T(S)] a^{\prime}$. These notions are discussed in detail in our prior work $[24,27]{ }^{4}$

To complete the definition, we need to specify the action of the functors on morphisms and show that they constitute PG-functors. The action on morphisms can be uniquely reconstructed from the action on edges because, if $F$ is a PGfunctor, then $F(\langle f\rangle)=\langle F(f)\rangle$, i.e., $F(\langle f\rangle)$ is the graph of a strict-continuous function. There is evidently at most one such function. We exhibit these functions for the functors involved in the interpretation of IA+:

- $\operatorname{Comm}(f)=\tau_{f}$, which is strict and continuous by definition.

$-\operatorname{ExP}_{\delta}(f)=\left[\phi_{f} \rightarrow \mathrm{id}_{\llbracket \delta \rrbracket}\right]$ sends an expression valuation $e \in \operatorname{ExP}_{\delta}(X)$ to $e \circ \phi_{f} \in \operatorname{ExP}_{\delta}(Y)$, which is evidently strict and continuous.

- $(F \times G)(f)=F(f) \times G(f)$, which preserves strictness and continuity.

- $(F \Rightarrow G)\left(f: X \rightarrow X^{\prime}\right)$ sends a family $\left\{t_{h} \in[F(Z) \rightarrow G(Z)]\right\}_{h: Z \leftarrow X}$ to the corresponding family $\left\{t_{\left(h^{\prime} ; f\right)}\right\}_{h^{\prime}: Z \leftarrow X^{\prime}}$, which is evidently strict and continuous.

- $(\operatorname{CLS} F)\left(f: X \rightarrow X^{\prime}\right)$ is just the identity morphism $\operatorname{id}_{(\mathrm{CLS} F)(X)}$.

Using these functor actions, we can upgrade any value $d$ of type $\theta$ at world $X$ to a future world $Y$. When the morphism $f: Y \rightarrow X$ is clear from the context, we often use the short-hand notation $d \uparrow_{X}^{Y} \triangleq \llbracket \theta \rrbracket(f)(d)$ to denote such upgrading.

\section{Interpretation of terms}

The meaning of a term $M$ with typing: $x_{1}: \theta_{1}, \ldots, x_{n}: \theta_{n} \vdash M: \theta$ is a parametric transformation of type

$$
\llbracket M \rrbracket:\left(\prod_{x_{i}} \llbracket \theta_{i} \rrbracket\right) \rightarrow \llbracket \theta \rrbracket
$$

This means that, for each world (Reynolds monoid) $X, \llbracket M \rrbracket_{X}$ is a continuous function of type $\left(\prod_{x_{i}} \llbracket \theta_{i} \rrbracket(X)\right) \rightarrow \llbracket \theta \rrbracket(X)$ such that all relations are preserved, i.e., for any relation $R: X \leftrightarrow X^{\prime}$, the meanings $\llbracket M \rrbracket_{X}$ and $\llbracket M \rrbracket_{X^{\prime}}$ are related by $\left(\prod_{x_{i}} \llbracket \theta_{i} \rrbracket(R)\right) \rightarrow \llbracket \theta \rrbracket(R)$. To the extent that IA + is a simply typed lambda calculus, this is standard $[9,18]$. We show the basic constructs:

$$
\begin{aligned}
& \llbracket x \rrbracket_{X}(u)=u(x) \\
& \llbracket \lambda x: \theta \cdot M \rrbracket_{X}(u)=\Lambda h: Z \leftarrow X \cdot \lambda d: \llbracket \theta \rrbracket(Z) . \llbracket M \rrbracket_{Z}\left(u \uparrow_{X}^{Z}[x \mapsto d]\right) \\
& \llbracket M N \rrbracket_{X}(u)=\llbracket M \rrbracket_{X}(u)\left[\operatorname{id}_{X}: X \rightarrow X\right]\left(\llbracket N \rrbracket_{X}(u)\right)
\end{aligned}
$$

\footnotetext{
${ }^{4}$ We are glossing over some detail here because constructing colimits of cpo's is a nontrivial exercise [12]. We prefer the alternative solution outlined in [24], where CPO's are coupled with partial equivalence relations to define more manageable colimits.
} 
The parameter $u$ may be thought of as an "environment" that provides values for the free identifiers, specifically in the given world $X$. The meaning of a lambda abstraction of type $\theta \rightarrow \theta^{\prime}$ is in $\left(\llbracket \theta \rrbracket \Rightarrow \theta^{\prime}\right)(X)$, which consists of families of the form $\left\{t_{h}\right\}_{h: Z \leftarrow X}$. Here, we are using notation " $\Lambda h: Z \leftarrow X$ " borrowed from the polymorphic lambda calculus to express the $h$ parameter. Note that the body of the abstraction is interpreted in the future world $Z$ and the environment $u$ is upgraded to this world. Parametricity in $Z$ is crucial for capturing the fact that $\llbracket M \rrbracket_{Z}$ does not directly access any information of the future world. In the interpretation of function application terms, we are again using the polymorphic lambda calculus notation to pass in the $h$ parameter, viz., $\operatorname{id}_{X}: X \rightarrow X$.

The interpretation of class definitions is given by:

$\llbracket$ class : $\theta$ local $C x$ init $A$ meth $M \rrbracket_{X}(u)=\left\langle Z,\left(\llbracket A \rrbracket_{Z}\left(u_{0}\right)\right)^{*}\left(z_{0}\right), \llbracket M \rrbracket_{Z}\left(u_{0}\right) \downarrow\right.$ where $\left\langle Z,\left(z_{0}, m\right)\right\rangle=\llbracket C \rrbracket_{X}(u)$ and $u_{0}=\{x \mapsto m\}$

This says that the package for the class $C$ is opened and a new package for the class term is created using it. We are depending on the fact that the class definition is a closed term. So, the only free identifier in $A$ and $M$ is $x$.

The interpretation of the new construct for creating class instances is:

$$
\begin{gathered}
\llbracket \text { new } C \text { o. } P \rrbracket_{X}(u)=\left(\lambda s .\left[s, z_{0}\right]_{\perp}\right) \cdot \llbracket P \rrbracket_{X \star Z}\left(u \uparrow_{X}^{X \star Z}\left[o \rightarrow m_{Z} \uparrow_{Z}^{X \star Z}\right]\right) \cdot(\lambda(s, z) . s) \\
\text { where }\left\langle Z,\left(z_{0}, m\right)\right\rangle=\llbracket C \rrbracket_{X}(u)
\end{gathered}
$$

The interpretation extends the current world $X$ to $X \star Z$, where $Z$ is a store for the internal state of the class, and executes the body of the new operator $(P)$ in the extended store. This execution is bracketed with an allocation and deallocation of the class instance, so that the overall command is still in the world $X$.

The interpretations of the primitives (constants) of IA+ is shown in Fig. 2. (Recall that the notation $f^{*}$ extends a function $f: A \rightarrow B$ to the type $A_{\perp} \rightarrow$ $B_{\perp}$.) The primitive $\operatorname{Var}[\delta]$ requires some explanation. Variables are treated in Idealized Algol as "objects" with methods for reading and writing their values (of types $\operatorname{ExP}_{\delta}$ and $\llbracket \delta \rrbracket \rightarrow$ COMM respectively). We use the shorthand $\operatorname{VAR}_{\delta}=$ $\operatorname{ExP}_{\delta} \times(\llbracket \delta \rrbracket \rightarrow$ Comm $)$ for the type of variables. In the world $V=(\llbracket \delta \rrbracket, T(\llbracket \delta \rrbracket))$, we can define a value mkvar that uses the states of the world $V$ to construct the two methods. The constant init $t_{\delta}$ represents some global value that is presumed to be used as the initial value for variables of type $\delta$.

To give additional insight, we also show a primitive called newvar, which has the effect of new $\operatorname{Var}[\delta]$. Given any world $X$, we have the expanded world $X \star V$ with injections $\iota_{1}: X \rightarrow X \star V$ and $\iota_{2}: V \rightarrow X \star V$ and $\operatorname{mkvar}_{V}{ }_{V}^{X \star V}=$ $\operatorname{VAR}_{\delta}\left(\iota_{2}\right)($ mkvar $) \in \operatorname{VAR}_{\delta}(X \star V)$. This variable object is provided as the argument to $p$. The remaining steps of newvar $X$ are the allocation and the deallocation of the local variable.

Lemma 2. All the combinators of Idealized Algol are parametric transformations.

Theorem 1. The meaning of every IA + term $x_{1}: \theta_{1}, \ldots, x_{n}:$ thet $a_{n} \vdash M: \theta$ is a parametric transformation of type $\left(\prod_{x_{i}} \llbracket \theta_{i} \rrbracket\right) \rightarrow \llbracket \theta \rrbracket$. 


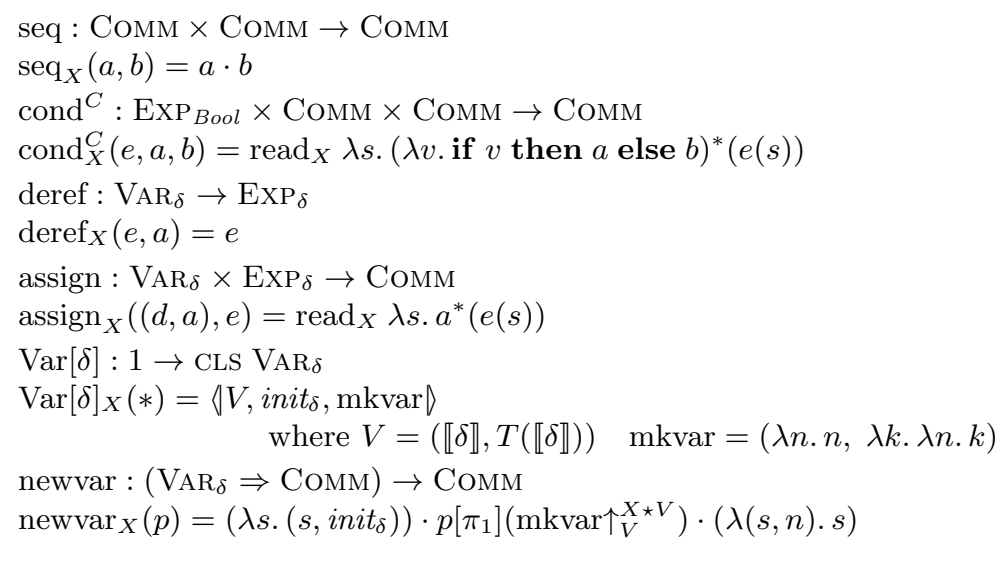

Fig. 2. Primitive operators of IA+

This completes the semantic definition of IA+.

\section{Example equivalences}

Meyer and Sieber [13] popularized the idea of demonstrating the efficacy of semantic models by proving test equivalences. All the equivalences discussed in their paper hold in our model. The first example below was left open by them. It can be proved using the state-based parametricity model in [18] as well as the event-based model $[14,22]$. We use it here for illustrative purposes.

Example 1. We can define two classes for counter objects as follows:
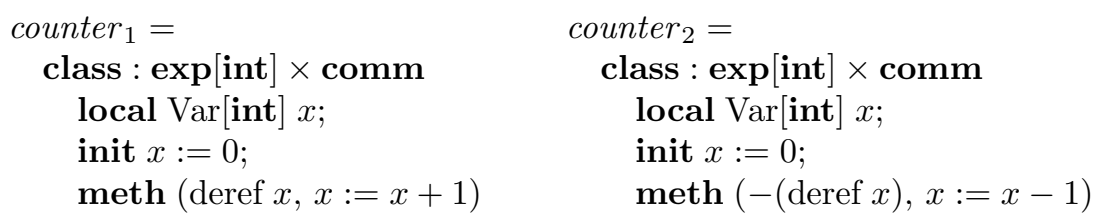

Their meanings should be semantic values of type:

$$
\exists_{Z}\left(\mathcal{Q}_{Z}\right)_{\perp} \times\left(\operatorname{ExP}_{\text {Int }} \times \operatorname{CoMM}\right)(Z)
$$

The meaning of the class counter $r_{1}$ is as follows: 
- The store $Z_{1}$ for the object is given by ${ }^{5}$

$$
\begin{aligned}
& \mathcal{Q}_{Z_{1}}=\text { Int } \\
& \mathcal{T}_{Z_{1}}=\text { read-closure of }\{I\} \cup\{\operatorname{inc}(k) \mid k \geq 0\} \\
& \quad \text { where } \operatorname{inc}(k)=\lambda n . n+k
\end{aligned}
$$

Note that $\mathcal{T}_{Z_{1}}$ is a monoid with the unit element inc $(0)$.

- The initial value is 0 .

- The method suite in $\left(\operatorname{ExP}_{I n t} \times \operatorname{Comm}\right)\left(Z_{1}\right)$ is the pair:

$$
\operatorname{meth}_{1}=((\lambda n . n), \operatorname{inc}(1))
$$

The meaning of the class counter $_{2}$ is similar:

- The store $Z_{2}$ for the object is given by

$$
\begin{aligned}
& \mathcal{Q}_{Z_{2}}=\text { Int } \\
& \mathcal{T}_{Z_{2}}=\text { read-closure of }\{\bar{I}\} \cup\{\operatorname{dec}(k) \mid k \geq 0\} \\
& \quad \text { where } \operatorname{dec}(k)=\lambda n . n-k
\end{aligned}
$$

- The initial value is 0 .

- The method suite in $\left(\operatorname{ExP}_{\text {Int }} \times \operatorname{ComM}\right)\left(Z_{2}\right)$ is the pair:

$$
\text { meth }_{2}=((\lambda n .-n), \operatorname{dec}(1))
$$

To demonstrate that the two classes are equal in the parametric colimit, we can exhibit a relation $R: Z_{1} \leftrightarrow Z_{2}$ that is preserved by the initialization and the method suite. The relation is $S: Z_{1} \leftrightarrow Z_{2}$, given by: ${ }^{6}$

$$
\begin{aligned}
& S_{Q}=\{(n,-n) \mid n \geq 0\} \\
& S_{T}=\{(\bar{\perp}, \bar{\perp})\} \cup\{(\operatorname{inc}(k), \operatorname{dec}(k)) \mid k \geq 0\}
\end{aligned}
$$

The preservation properties to be verified are:

$$
\begin{aligned}
& 0\left[\left(S_{Q}\right)_{\perp}\right] 0 \\
& \operatorname{meth}_{1}\left[\left(\operatorname{ExP}_{\text {Int }} \times \operatorname{COMM}\right)(S)\right] \text { meth }_{2}
\end{aligned}
$$

It is easy to verify them once we note that $\left(\operatorname{ExP}_{\text {Int }} \times \operatorname{Comm}\right)(S)=\left[S_{Q} \rightarrow\right.$ $\left.\Delta_{\text {Int }}\right] \times S_{T}$.

\footnotetext{
${ }^{5}$ Since the class is defined using a variable object, the semantic definition states the meaning in terms of the world $V$ for the internal state of the counter, which includes the full transformation monoid $(\llbracket \delta \rrbracket, T(\llbracket \delta \rrbracket))$. However, the meaning of the class is an abstract "package," unique up to behavioral equivalence. So, we can cut down the transformation component of the world to just those transformations directly used in the class via behavioral equivalence.

${ }^{6}$ For ease of exposition, we ignore the read-closure condition of the transformation relations.
} 
Example 2 (Pitts and Stark "awkward" example). Consider the following classes:

$$
\begin{array}{cc}
C_{1}=\text { class }: \text { comm } \rightarrow \text { comm } & C_{2}=\text { class }: \text { comm } \rightarrow \text { comm } \\
\text { local } \operatorname{Var}[\text { int }] x ; & \text { local } \operatorname{Var}[\text { int }] x ; \\
\text { init } x:=0 ; & \text { init } x:=0 ; \\
\text { meth } \lambda c . x:=1 ; c ; \text { test }(x=1) & \text { meth } \lambda c . c
\end{array}
$$

where test $(b)=$ if $b$ then skip else diverge.

A relation $S$ between the internal states of the classes $C_{1}$ and $C_{2}$ has two components, a relation $S_{Q}$ between their state sets and a relation $S_{T}$ between their state transformations. The transformation component $S_{T}$ relates the transformations null and put(1) of $C_{1}$ to the null transformation of $C_{2}$. Since the $c$ arguments to the methods are assumed to be related by $S_{T}$, we can conclude that the call to $c$ in $C_{1}$ executes some combination of null and put(1) actions, with the result that $x$ is 1 after the call.

We show the detailed proof. The meanings of the classes should be semantic values of type:

$$
\exists_{Z}\left(\left(\mathcal{Q}_{Z}\right)_{\perp} \times \forall_{g: Y \rightarrow Z} \operatorname{Comm}(Y) \rightarrow \operatorname{Comm}(Y)\right)
$$

The meaning of the class $C_{1}$ is as follows:

$$
\begin{aligned}
& \mathcal{Q}_{Z_{1}}=\text { Int } \\
& \mathcal{T}_{Z_{1}}=\text { read-closure of }\left\{I, \operatorname{null} Z_{2}, \operatorname{put}(1)\right\} \\
& \text { init }_{1}=0 \\
& \text { meth }_{1}=\Lambda g: Z_{1} \leftarrow Y \cdot \lambda c: \operatorname{Comm}(Y) . \\
& \quad \operatorname{put}(1) \uparrow Y Z_{1} \cdot c \cdot \operatorname{check}(1) \uparrow Y_{Z_{1}}
\end{aligned}
$$

where put $(k)=\lambda n . k$ and $\operatorname{check}(k)=\operatorname{read} \lambda n$. if $n=k$ then null else $\bar{\perp}$.

The meaning of the class $C_{2}$ is similar:

$$
\begin{aligned}
& \mathcal{Q}_{Z_{2}}=\text { Int } \\
& \mathcal{T}_{Z_{2}}=\text { read-closure of }\left\{\bar{\perp}, \operatorname{null}_{Z_{2}}\right\} \\
& \text { init }_{2}=0 \\
& \text { meth } h_{2}=\Lambda g: Z_{2} \leftarrow Y \cdot \lambda c: \operatorname{Comm}(Y) \cdot c
\end{aligned}
$$

To demonstrate that the two classes are equal, we exhibit a relation $S: Z_{1} \leftrightarrow$ $Z_{2}$ given by:

$$
\begin{aligned}
& S_{Q}=\{(n, 0) \mid n \geq 0\} \\
& S_{T}=\left\{(\bar{\perp}, \bar{\perp}),\left(\operatorname{null}_{Z_{1}}, \operatorname{null}_{Z_{2}}\right),\left(\operatorname{put}(1), \operatorname{null}_{Z_{2}}\right)\right\}
\end{aligned}
$$

The preservation properties to be verified are:

$$
\begin{aligned}
& \text { init }_{1}\left[\left(S_{Q}\right)_{\perp}\right] \text { init }_{2} \\
& \text { meth }_{1}[(\operatorname{ComM} \Rightarrow \operatorname{CoMM})(S)] \text { meth }_{2}
\end{aligned}
$$


Note that $(\mathrm{Comm} \Rightarrow \mathrm{Comm}) S=\forall_{R \rightarrow S} \operatorname{Comm}(R) \rightarrow \operatorname{Comm}(R)=\forall_{R \rightarrow S} R_{T} \rightarrow$ $R_{T}$. So, the relationship to be proved between the two method suites is:

$$
\begin{gathered}
\forall g_{1}: Y_{1} \rightarrow Z_{1} \cdot \forall g_{2}: Y_{2} \rightarrow Z_{2} \cdot g_{1}[R \rightarrow S] g_{2} \Longrightarrow \\
\forall c_{1}, c_{2} \cdot c_{1}\left[R_{T}\right] c_{2} \Longrightarrow \\
\text { meth }_{1}\left[g_{1}\right]\left(c_{1}\right)\left[R_{T}\right] \operatorname{meth}_{2}\left[g_{2}\right]\left(c_{2}\right)
\end{gathered}
$$

Since put(1) $\left[S_{T}\right] \operatorname{null}_{Z_{1}}$, we have put(1) $\uparrow_{Z_{1}}^{Y_{1}}\left[R_{T}\right] \operatorname{null} \uparrow_{Z_{2}}^{Y_{2}}$. Since $c_{1}\left[R_{T}\right] c_{2}$ by assumption, the state in $Z_{1}$ (the value of $x$ ) is 1 , as argued above. Therefore check(1) has the effect of null $Z_{1}$. Hence, we have the required property.

Example 3 (Dreyer, Neis and Birkedal). Consider the following classes:

$$
\begin{array}{cc}
C_{1}=\text { class }: \text { comm } \rightarrow \text { comm } & C_{2}=\text { class }: \text { comm } \rightarrow \text { comm } \\
\text { local } \operatorname{Var}[\mathbf{i n t}] x ; & \text { local } \operatorname{Var}[\mathbf{i n t}] x ; \\
\text { init } x:=0 ; & \text { init } x:=0 ; \\
\text { meth } \lambda c . x:=0 ; c ; x:=1 ; c ; \text { test }(x=1) & \text { meth } \lambda c . c ; c
\end{array}
$$

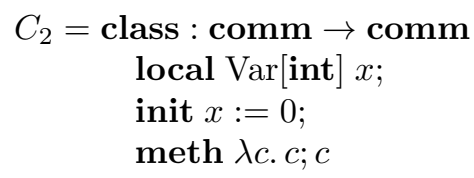

where test $(b)=$ if $b$ then skip else diverge.

This example is similar to the "awkward" example, except that we have two calls to $c$ in the method of $C_{1}$, interspersed by different assignments to $x$. The differences from the above example are as follows:

$$
\begin{aligned}
\mathcal{T}_{Z_{1}}= & \text { read-closure of }\left\{\bar{\perp}, \operatorname{null} Z_{Z_{1}}, \operatorname{put}(0), \operatorname{put}(1)\right\} \\
\text { meth }_{1}= & \Lambda g: Z_{1} \leftarrow Y \cdot \lambda c: \operatorname{ComM}(Y) \cdot \\
& \quad \operatorname{put}(0) \uparrow_{Z_{1}}^{Y} \cdot c \cdot \operatorname{put}(1) \uparrow_{Z_{1}}^{Y} \cdot c \cdot \operatorname{check}(1) \uparrow_{Z_{1}}^{Y} \\
\mathcal{T}_{Z_{2}}= & \operatorname{read}-\operatorname{closure} \text { of }\left\{\bar{\perp}, \operatorname{null} Z_{Z_{2}}\right\} \\
\text { meth }_{2}= & \Lambda g: Z_{2} \leftarrow Y \cdot \lambda c: \operatorname{ComM}(Y) \cdot c \cdot c \\
S_{T}= & \left\{(\bar{\perp}, \bar{\perp}),\left(\operatorname{null}_{Z_{1}}, \operatorname{null}_{Z_{2}}\right),\left(\operatorname{put}(1), \operatorname{null}_{Z_{2}}\right)\right\}
\end{aligned}
$$

It is worth noting that the relation $S_{T}: \mathcal{T}_{Z_{1}} \leftrightarrow \mathcal{T}_{Z_{2}}$ is the same as that in the awkward example.

We verify the simulation property

$$
\text { meth }_{1}[(\text { Cомм } \Rightarrow \text { COMM })(S)] \text { meth }_{2}
$$

as in the previous example, which involves the condition:

$$
\begin{gathered}
\forall g_{1}: Y_{1} \rightarrow Z_{1} \cdot \forall g_{2}: Y_{2} \rightarrow Z_{2} \cdot g_{1}[R \rightarrow S] g_{2} \Longrightarrow \\
\forall c_{1}, c_{2} \cdot c_{1}\left[R_{T}\right] c_{2} \Longrightarrow \\
\text { meth }_{1}\left[g_{1}\right]\left(c_{1}\right)\left[R_{T}\right] \operatorname{meth}_{2}\left[g_{2}\right]\left(c_{2}\right)
\end{gathered}
$$

We first argue that meth $\left[g_{1}\right]\left(c_{1}\right)$ and meth $_{2}\left[g_{2}\right]\left(c_{2}\right)$ are related by $R_{Q} \rightarrow R_{Q}$. Starting from related initial states $n$ and 0 , the first action in meth $h_{1}$ is put(0), which changes the local state to 0 . Calling $c$ has the effect of either null $Z_{Z_{1}}$ or $\operatorname{put}(1)$ on $x$. So, $x$ is either 0 or 1 , both of which are related to 0 by $R_{Q}$. The next action put(1) overrides the previous effect and changes the local state to 
1. The second call to $c$ again has the effect of either null $Z_{1}$ or put(1), with the result that the local state continues to be 1 and, so, check(1) succeeds. Thus, the overall effect of meth $_{1}$ is to set the local state to 1, i.e., a put(1) action, and two calls to $c$ for the effects on the non-local state. This is related to $c \cdot c$ in $m^{2} t h_{2}$ by the $R_{T}$ relation.

Dreyer et al. [6] characterize actions as put(0) in meth $_{1}$ as "private transitions" because they are not visible at the end of method calls. Note that no special treatment is needed in the semantics to capture such private transitions. Essentially, the private transitions are handled by $R_{Q}$, the state components of the Reynolds monoid-relations, whereas the public transitions are handled by $R_{T}$, the transformation components of the relations.

Even though all our examples contain a single instance variable of $\operatorname{Var}[\delta]$ class, we should point out that the semantic methods are not restricted to such cases. See [27] for examples with more intricate instance variable structures where similar methods are applied.

\section{Conclusion}

We have outlined a new denotational semantic model for class-based Algol-like languages, which combines the advantages of the existing models. Similar to the state-based models, it is able to represent the effect of operations as state transformations. At the same time, it also represents stores as rudimentary form of objects, whose state changes are treated from the outside in a modular fashion. Further, this modeling allows one to prove observational equivalences of programs that were not possible in the previous models. This work complements that of Ahmed, Dreyer and colleagues $[3,6]$ who use an operational approach to develop similar reasoning principles.

In principle, this work could have been done any time after 1983, because Reynolds used a similar framework for his semantics in [30] and formulated relational parametricity in [32]. We can only speculate why it wasn't done. The alternative model invented by Oles [20] was considered equivalent to the Reynolds's model and it appeared to be simpler as well as more general. However, sharp differences between the two models become visible as soon as relational parametricity is considered. This fact was perhaps not appreciated in the intervening years.

In terms of further work to be carried out, we have not addressed the issues of dynamic storage (pointers) but we expect that the prior work in parametricity semantics [29] will be applicable. We have not considered higher-order store, i.e., storing procedures in variables. This problem is known to be hard in the framework of functor category models and it may take some time to get resolved. More exciting work awaits to be done in applying these ideas to study program reasoning, including Specification Logic [31,33], Separation Logic, Rely-guarantee and Deny-guarantee reasoning techniques [5, 36]. 


\section{Bibliography}

[1] S. Abramsky, K. Honda, and G. McCusker. A fully abstract game semantics for general references. In LICS 1998, pages 334-344, 1998.

[2] S. Abramsky and G. McCusker. Linearity, sharing and state. In Algol-like Languages O'Hearn and Tennent [19], chapter 20.

[3] A. Ahmed, D. Dreyer, and A. Rossberg. State-dependent representation independence. In Thirty Sixth Ann. ACM Symp. on Princ. of Program. Lang. ACM, 2009.

[4] T. Dinsdale-Young, M. Dodds, P. Gardner, M. J. Parkinson, and V. Vafeiadis. Concurrent abstract predicates. In European Conf. on ObjectOriented Programming - ECOOP, pages 504-528, Berlin, 2010. SpringerVerlag.

[5] M. Dodds, M. Feng, M. Parkinson, and V. Vafeiadis. Deny-guarantee reasoning. In European Symposium on Programming, pages 363-377, Berlin, 2009. Springer-Verlag.

[6] D. Dreyer, G. Neis, and L. Birkedal. The impact of higher-order state and control effects on local relational reasoning. In ICFP, 2010.

[7] D. Dreyer, G. Neis, A. Rossberg, and L. Birkedal. A relational modal logic for higher-order stateful ADTs. In Thirty Seventh Ann. ACM Symp. on Princ. of Program. Lang. ACM, 2010.

[8] B. P. Dunphy. Parametricity as a Notion of Uniformity in Reflexive Graphs. $\mathrm{PhD}$ thesis, University of Illinois, Dep. of Mathematics, 2002. available electronically from http://www.cs.bham.ac.uk/ udr.

[9] B. P. Dunphy and U. S. Reddy. Parametric limits. In Proc. 19th Ann. IEEE Symp. on Logic in Comp. Sci., pages 242-253. IEEE, July 2004.

[10] S. Eilenberg. Automata, Languages, and Machines. Academic Press, 1974. (Volumes A and B).

[11] W. M. L. Holcombe. Algebraic Automata Theory. Cambridge Studies in Advanced Mathematics. Cambridge Univ. Press, Cambridge, 1982.

[12] A. Jung. Colimits in DCPO. Unpublished Manuscript, 1990.

[13] A. R. Meyer and K. Sieber. Towards fully abstract semantics for local variables. In Fifteenth Ann. ACM Symp. on Princ. of Program. Lang., pages 191-203. ACM, 1988. (Reprinted as Chapter 7 of [19]).

[14] P. W. O'Hearn and U. S. Reddy. Objects, interference and the Yoneda embedding. In S. D. Brookes, M. Main, A. Melton, and M. Mislove, editors, Math. Found. of Program. Semantics: Eleventh Ann. Conference, volume 1 of Elect. Notes in Theor. Comput. Sci. Elsevier, 1995.

[15] P. W. O'Hearn and U. S. Reddy. Objects, interference and the Yoneda embedding. Theoretical Computer Science, 228(1):211-252, 1999.

[16] P. W. O'Hearn and J. C. Reynolds. From Algol to polymorphic linear lambda-calculus. J. ACM, 47(1):167-223, Jan 2000. 
[17] P. W. O'Hearn and R. D. Tennent. Semantics of local variables. In M. P. Fourman, P. T. Johnstone, and A. M. Pitts, editors, Applications of Categories in Computer Science, pages 217-238. Cambridge Univ. Press, 1992.

[18] P. W. O'Hearn and R. D. Tennent. Parametricity and local variables. J. ACM, 42(3):658-709, 1995. (Reprinted as Chapter 16 of [19]).

[19] P. W. O'Hearn and R. D. Tennent. Algol-like Languages (Two volumes). Birkhäuser, Boston, 1997.

[20] F. J. Oles. Type algebras, functor categories and block structure. In M. Nivat and J. C. Reynolds, editors, Algebraic Methods in Semantics, pages 543-573. Cambridge Univ. Press, 1985.

[21] A. M. Pitts and I. D. B. Stark. Operational reasoning for functions with local state. In A. M. Gordon and A. M. Pitts, editors, Higher Order Operational Techniques in Semantics, pages 227-274. Cambridge Univ. Press, Cambridge, 1998.

[22] U. S. Reddy. Global state considered unnecessary: An introduction to object-based semantics. J. Lisp and Symbolic Computation, 9:7-76, 1996. (Reprinted as Chapter 19 of [19]).

[23] U. S. Reddy. When parametricity implies naturality. Electronic manuscript, University of Birmingham, http://www.cs.bham.ac.uk/ udr/, July 1997.

[24] U. S. Reddy. Objects and classes in Algol-like languages. In FOOL 5: Fifth Intern. Workshop on Foundations of Object-oriented Languages. electronic

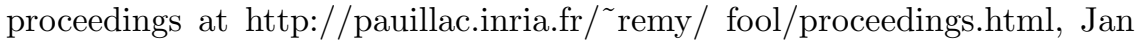
1998.

[25] U. S. Reddy. Parametricity and naturality in the semantics of Algol. Talk at the Intern. Workshop on Math. Found. of Program. Semantics, London, May 1998.

[26] U. S. Reddy. Parametricity and naturality in the semantics of Algollike languages. Electronic manuscript, University of Birmingham, http://www.cs.bham.ac.uk/ udr/, Dec 1998.

[27] U. S. Reddy. Objects and classes in Algol-like languages. Information and Computation, 172:63-97, 2002.

[28] U. S. Reddy. An automata-theoretic model of objects. In E. Zucca, editor, 2011 Intl. Workshop on Foundations of Object-Oriented Languages. electronic proceedings at http://www.disi.unige.it/person/ZuccaE/FOOL2011/, 2011.

[29] U. S. Reddy and H. Yang. Correctness of data representations involving heap data structures. Science of Computer Programming, 50(1-3):129-160, Mar 2004.

[30] J. C. Reynolds. The essence of Algol. In J. W. de Bakker and J. C. van Vliet, editors, Algorithmic Languages, pages 345-372. North-Holland, 1981. (Reprinted as Chapter 3 of [19]).

[31] J. C. Reynolds. Idealized Algol and its specification logic. In D. Neel, editor, Tools and Notions for Program Construction, pages 121-161. Cambridge Univ. Press, 1982. (Reprinted as Chapter 6 of [19]).

[32] J. C. Reynolds. Types, abstraction and parametric polymorphism. In R. E. A. Mason, editor, Information Processing '83, pages 513-523. NorthHolland, Amsterdam, 1983. 
[33] R. D. Tennent. Semantical analysis of specification logic. Inf. Comput., 85 (2):135-162, 1990. (Reprinted as Chapter 13 of [19]).

[34] R. D. Tennent. Semantics of Programming Languages. Prentice-Hall International, London, 1991.

[35] R. D. Tennent. Denotational semantics. In S. Abramsky, D. M. Gabbay, and T. S. E. Maibaum, editors, Handbook of Logic in Computer Science, volume 3, pages 169-322. Oxford University Press, 1994.

[36] V. Vafeiadis and M. Parkinson. A marriage of rely/guarantee and Separation Logic. In CONCUR 2007, volume 4703 of $L N C S$, pages 256-271, 2007. 


\section{Appendix}

\section{A Definitions}

\section{B Proofs}

\section{Proof of Lemma 1}

$\mathbf{R M}$ is evidently relational. For op-fibration, we need a weakest pre-edge $\left[f, f^{\prime}\right] R$ for every $R, f$ and $f^{\prime}$ as in the situation shown below:

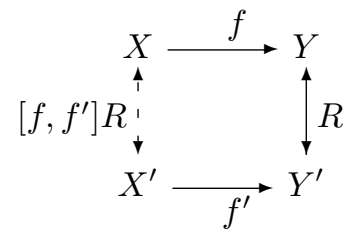

We define it as the pair

$$
\left[f, f^{\prime}\right] R=\left(R_{Q}\left[\phi_{f}, \phi_{f^{\prime}}\right],\left[\tau_{f}, \tau_{f^{\prime}}\right] R_{T}\right)
$$

Diagrammatically:

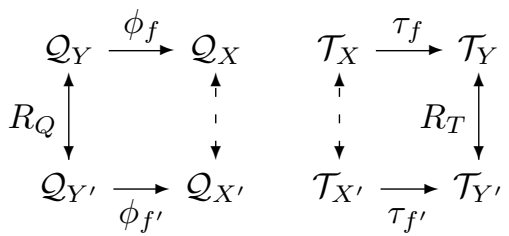

The first component is the strongest post-relation in Set which is nothing but the "direct image":

$$
\begin{aligned}
R_{Q}\left[\phi_{f}, \phi_{f^{\prime}}\right]=\left\{\left(x, x^{\prime}\right) \mid \exists y, y^{\prime} \cdot y\left[R_{Q}\right] y^{\prime}\right. & \wedge \\
\phi_{f}(y)=x & \left.\wedge \phi_{f^{\prime}}\left(y^{\prime}\right)=x^{\prime}\right\}
\end{aligned}
$$

and the second component is the weakest pre-relation in the reflexive graph of complete ordered monoids which is nothing but the "inverse image":

$$
\left[\tau_{f}, \tau_{f^{\prime}}\right] R_{T}=\left\{\left(a, a^{\prime}\right) \mid \tau_{f}(a)\left[R_{T}\right] \tau_{f^{\prime}}\left(a^{\prime}\right)\right\}
$$

\section{Proof of Lemma 2}

We show selected cases. For the assignment operation, let $(d, a)\left[\operatorname{VAR}_{\delta}(R)\right]$ $\left(d^{\prime}, a^{\prime}\right)$ and $e\left[\operatorname{Exp}_{\delta}(R)\right] e^{\prime}$. Then

$$
\begin{gathered}
\left(\lambda s . a^{*}(e(s))\right)\left[R_{Q} \rightarrow R_{T}\right]\left(\lambda s^{\prime} \cdot a^{\prime *}\left(e^{\prime}\left(s^{\prime}\right)\right)\right) \\
\left(\operatorname{read}_{X} \lambda s . a^{*}(e(s))\right)\left[R_{T}\right]\left(\operatorname{read}_{X^{\prime}} \lambda s^{\prime} \cdot a^{\prime *}\left(e^{\prime}\left(s^{\prime}\right)\right)\right)
\end{gathered}
$$


The second step follows from the fact that the relations are compatible with the diagonal operation.

Consider the newvar combinator. Let $R: X \leftrightarrow X^{\prime}$ be a relation of rtm's and assume $p\left[\left(\operatorname{VAR}_{\delta} \Rightarrow \mathrm{COMM}\right)(R)\right] p^{\prime}$.

1. The relation $(\operatorname{VAR} \Rightarrow \operatorname{COMM})(R)$ is $\forall_{S \rightarrow R} \operatorname{VAR}(S) \rightarrow \operatorname{Comm}(S)$. In the particular case used in the combinator, $S$ is instantiated to $R \star I_{V}: X \star V \leftrightarrow X^{\prime} \star$ $V$. So, we obtain $p\left[\left(\pi_{1}\right)_{X, V}\right]\left[\operatorname{VAR}\left(R \star I_{V}\right) \rightarrow \operatorname{COMM}\left(R \star I_{V}\right)\right] p\left[\left(\pi_{1}\right)_{X^{\prime}, V}\right]$.

2. We argue that $\operatorname{mkvar} \uparrow_{V}^{X}$ and $\operatorname{mkvar} \uparrow_{V}^{X^{\prime}}$ are related by $\operatorname{VAR}_{\delta}\left(R \star I_{V}\right)$. Firstly, $\lambda(s, n) . n$ and $\lambda\left(s^{\prime}, n^{\prime}\right) . n^{\prime}$ are related by $\operatorname{ExP}_{\delta}\left(R \star I_{V}\right)$, i.e., $R_{Q} \times \Delta_{\llbracket \delta \rrbracket} \rightarrow \Delta_{\llbracket \delta \rrbracket}$. Secondly, $\lambda k \cdot \lambda(s, n) .(s, k)$ and $\lambda k^{\prime} . \lambda\left(s^{\prime}, n^{\prime}\right) \cdot\left(s^{\prime}, k^{\prime}\right)$ are related by $\Delta_{\llbracket \delta \rrbracket} \rightarrow$ $\operatorname{Comm}\left(R \star I_{V}\right)$, i.e., $\Delta_{\llbracket \delta \rrbracket} \rightarrow R_{T} \star \Delta_{T(\llbracket \delta \rrbracket)}$. Note that $\lambda(s, n) .(s, k)$ can be expressed as null ${ }_{X} \otimes(\lambda n . k)$ in $\mathcal{T}_{X} \star T(\llbracket \delta \rrbracket)$. null ${ }_{X}$ and null $_{X^{\prime}}$ are related by $\mathcal{T}_{X}$ and $\lambda n . k$ is related to itself by $\Delta_{T(\llbracket \delta \rrbracket)}$.

3. Therefore, $p\left[\left(\pi_{1}\right)_{X, V}\right]\left(\operatorname{mkvar} \uparrow_{V}^{X}\right)\left[\operatorname{COMM}\left(R \star I_{V}\right)\right] p\left[\left(\pi_{1}\right)_{X^{\prime}, V}\right]\left(\operatorname{mkvar\uparrow } X_{V}^{X^{\prime}}\right)$.

4. The relation $\operatorname{Comm}\left(R \star I_{V}\right)$ is $R_{T} \star\left(I_{V}\right)_{T}$ where $\left(I_{V}\right)_{T}=\left[\Delta_{\llbracket \delta \rrbracket} \rightarrow \Delta_{\llbracket \delta \rrbracket}\right]$. So, the instances $t=p\left[\left(\pi_{1}\right)\right]\left(\operatorname{mkvar} \uparrow_{V}^{X}\right)$ and $t^{\prime}=p^{\prime}\left[\left(\pi_{1}\right)\right]\left(\operatorname{mkvar} \uparrow_{V}^{X^{\prime}}\right)$ are related by $R_{T} \star\left[\Delta_{\llbracket \delta \rrbracket} \rightarrow \Delta_{\llbracket \delta \rrbracket}\right]$. So, for any $s \in \mathcal{Q}_{X}, s^{\prime} \in \mathcal{Q}_{X^{\prime}}$ and $n \in \llbracket \delta \rrbracket$, there exist $a_{s n}, a_{s^{\prime} n}^{\prime}, b_{n}$ such that $a_{s n}[R] a_{s^{\prime} n}^{\prime}, t(s, n)=\left(a_{s n} \otimes b_{n}\right)(s, n)$ and $t^{\prime}\left(s^{\prime}, n\right)=\left(a_{s^{\prime} n}^{\prime} \otimes b_{n}\right)\left(s^{\prime}, n\right)$.

5. In particular, the above statement holds for $n=0$. So, unless $b_{0}(0)=\perp$, $(t \cdot(\lambda(s, n) . s))(s, 0)=a_{s 0}(s)$ and $\left(t^{\prime} \cdot\left(\lambda\left(s^{\prime}, n\right) . s^{\prime}\right)\right)\left(s^{\prime}, 0\right)=a_{s^{\prime} 0}^{\prime}\left(s^{\prime}\right)$. In other words, newvar $\operatorname{nov}_{X}(p)(s)=a_{s 0}(s)$ and newvar $X^{\prime}\left(p^{\prime}\right)\left(s^{\prime}\right)=a_{s^{\prime} 0}^{\prime}\left(s^{\prime}\right)$. If, on the other hand, $b_{0}(0)=\perp$, both the functions evaluate to $\perp$. Hence, we can write $\operatorname{newvar}_{X}(p)$ as $\left(\operatorname{read}_{X} \lambda s\right.$. if $b_{0}(0)=\perp$ then $\perp$ else $\left.a_{s 0}\right)$, and similarly for newvar $_{X^{\prime}}\left(p^{\prime}\right)$. These two transformations are related by $R_{T}$.

The case of cond ${ }^{C}$ illustrates how expression evaluations are embedded in commands. Again, let $R: X \leftrightarrow X^{\prime}$ be a relation of rtm's and assume $e\left[\operatorname{ExP}_{\delta}(R)\right]$ $e^{\prime}, a[\operatorname{Comm}(R)] a^{\prime}$ and $b[\operatorname{Comm}(R)] b^{\prime}$. To show that $\operatorname{cond}_{X}^{C}(e, a, b)[\operatorname{Comm}(R)]$ $\operatorname{cond}_{X^{\prime}}^{C}\left(e^{\prime}, a^{\prime}, b^{\prime}\right)$, we need to show that $p=\left(\lambda s .(\lambda k \text {. if } k \neq 0 \text { then } a \text { else } b)^{*}(e(s))\right)$ and $p^{\prime}=\left(\lambda s^{\prime}\right.$. $\left.\left(\lambda k^{\prime} \text {. if } k^{\prime} \neq 0 \text { then } a^{\prime} \text { else } b^{\prime}\right)^{*}\left(e^{\prime}\left(s^{\prime}\right)\right)\right)$ are related by $R_{Q} \rightarrow R_{T}$. So, consider the action of the functions on states $s$ and $s^{\prime}$ such that $s[R] s^{\prime}$.

1. Since $\operatorname{Exp}_{\delta}(R)=\left[R \rightarrow \Delta_{\llbracket \delta \rrbracket}\right]$, we have $e(s)\left[\Delta_{\left.\llbracket \delta \rrbracket_{\perp}\right]} e^{\prime}\left(s^{\prime}\right)\right.$, i.e., $e(s)=e^{\prime}\left(s^{\prime}\right)$.

2. If $e(s)=e^{\prime}\left(s^{\prime}\right)=\perp$ then $p(s)=\perp_{\mathcal{T}_{X}}$ and $p^{\prime}\left(s^{\prime}\right) \stackrel{\perp=}{=} \mathcal{T}_{X^{\prime}}$, which are related $R_{T}$ since it is a pointed relation.

3. If $e(s)=e^{\prime}\left(s^{\prime}\right)=0$ then $p(s)=b$ and $p^{\prime}\left(s^{\prime}\right)=b^{\prime}$, which are related by $R_{T}$ by assumption that $b$ and $b^{\prime}$ are related by $\operatorname{Comm}(R)=R_{T}$. The case of $e(s)=e^{\prime}\left(s^{\prime}\right)$ being non-zero is similar.

All the other combinators can be similarly verified to be parametric.

\section{Treatment of general classes}

In the main body of the paper, we restricted attention to "constant classes" that have no free identifiers. Classes with free identifiers are quite useful, e.g., 
for defining nested classes. Here, we treat the general case. The interpretation of the general cls types is as follows:

$$
\begin{aligned}
(\operatorname{CLS} F)(X)= & \forall_{g: Y \rightarrow X} \exists_{h: Z \rightarrow Y} \\
& {\left[\mathcal{Q}_{Y} \rightarrow \mathcal{Q}_{Z}\right] \times F(Z) \times\left[Q_{Z} \rightarrow Q_{Y}\right] } \\
(\operatorname{CLS} F)(R)= & \forall P_{P \rightarrow R} \exists_{S \rightarrow P} \\
& {\left[P_{Q} \rightarrow S_{Q}\right] \times F(S) \times\left[S_{Q} \rightarrow P_{Q}\right] }
\end{aligned}
$$

The meaning of a class at world $X$ provides a way of creating instances at all future worlds $Y$, and such creation leads to a further future world $Z$. In addition to the method suite, of type $F(Z)$, we have allocation and deallocation operations, which are both irregular state transformations.

The notation $\exists_{h: Z \rightarrow X} T(Z)$ stands for an indexed "parametric colimit." It is a quotient of $\bigsqcup_{h: Z \rightarrow X} T(Z)$ under the transitive closure of the "similarity" relation $\sim$ defined by the rule:

$$
h\left[S \rightarrow I_{X}\right] h^{\prime} \wedge a[T(S)] a^{\prime} \Rightarrow\langle h, a\rangle \sim\left\langle h^{\prime}, a^{\prime}\right\rangle
$$

The equivalence class of $\langle h, a\rangle$ under $\sim^{*}$ is denoted by $\langle h, a\rangle$.

The functor action on morphisms is: (CLS $F)\left(f: X^{\prime} \rightarrow X\right)$ sends a family $\left\{t_{g}\right\}_{g: Y \rightarrow X}$ to the corresponding family $\left\{t_{\left(g^{\prime} ; f\right)}\right\}_{g^{\prime}: Y \rightarrow X^{\prime}}$.

The interpretation of new for such classes is:

$$
\begin{aligned}
& \llbracket \text { new } C \text { o. } P \rrbracket_{X}(u)=i \cdot \llbracket P \rrbracket_{Y}(h(u)[o \rightarrow m] \cdot d \\
& \quad \text { where } \backslash h: Y \rightarrow X, i, m, d\rangle=\llbracket C \rrbracket_{X}(u)\left[\operatorname{id}_{X}: X \rightarrow X\right]
\end{aligned}
$$

It makes use of the expansion morphism and the allocation and deallocation operations rather directly. 